\title{
Las políticas para la Sociedad de la Información y el Conocimiento en América Latina. Desde una mirada tecnologicista a un enfoque para el complejo
}

\author{
Ana Rivoir*
}

\begin{abstract}
Resumen
Desde hace más de una década, los países de América Latina se han sumado a los esfuerzos para facilitar la utilización de las Tecnologías de la Información y la Comunicación para el desarrollo. Comenzando por iniciativas en la educación en los ochenta y noventa hasta estrategias digitales actuales, se ha acumulado experiencia y revisión de la orientación de las políticas. A partir de estudios sobre la orientación de estas políticas es que constatamos que se ha producido un cambio en las mismas. En un inicio más tecnologicistas y orientadas por la centralidad de las TIC como impulsoras del bienestar y el desarrollo, hoy adquieren una mirada más compleja y sobre todo incluyendo las dimensiones sociales. ¿Se ha abandonado el paradigma dominante tecno-céntrico por otro más complejo? ¿Se refleja este cambio en la formulación y prioridades de las políticas?
\end{abstract}

Palabras clave: políticas/ sociedad de la información/TIC

\begin{abstract}
For more than a decade, Latin American countries have joined efforts to enable the use of Information Technology and Communication for development. By starting with education initiatives in the eighties and nineties to current digital strategies, experience and review of policy guidance has been accumulated. From studies on the orientation of these policies is that we find that there has been a change in them. They were initially more technological and guided by the centrality of ICT as a driving force of welfare and development; today they have acquired a more complex view and above all including social dimensions. Has the dominant techno-centric paradigm been abandoned to a more complex one? Is this change reflected in the formulation of policies and priorities?
\end{abstract}

Keywords: policy / information society / ICT

* Departamento de Sociología, Facultad de Ciencias Sociales, Universidad de la República Uruguay. Contacto: ana@fcs.edu.uy 


\section{Paradigmas de la Sociedad de la información en América Latina}

La Sociedad de la Información y el Conocimiento es motivo de distintas miradas enfoques y creencias. El momento histórico al que hace referencia este concepto tiene distintas denominaciones que dan cuenta de un debate acerca del alcance de las transformaciones experimentadas en las últimas décadas. Así encontramos denominaciones tales como Sociedad de la Información, Sociedad del Conocimiento, Sociedad del Aprendizaje y a su vez todas en plural a los efectos de marcar la diversidad de realidades.

Los procesos desencadenados, los proyectos de desarrollo en juego, las estrategias y la relación de poder entre los distintos actores, derivan en que se consolida un paradigma que presenta e impulsa a la SIC como una nueva modernización. Consolida supuestos que sustentan teorías y fundamentan visiones acerca de las TIC y su rol en el desarrollo de las personas y las sociedades, pues como señala Mattelart: “Las creencias de las que la noción de SI es portadora desencadenan fuerzas simbólicas que impulsan a actuar, a la vez que permiten actuar en un determinado sentido y no en otro." (Mattelart, 2002, p. 12)

América Latina está transitando un momento histórico de cambios políticos y transformaciones sociales. Las políticas están siendo revisadas y esto constituye un contexto adecuado para la actualización de viejas prácticas y para la innovación. La evidencia de que las políticas para la SIC hasta ahora implementadas, han tenido limitada incidencia en los procesos de desarrollo, es uno de los aspectos centrales a considerar. Se sostiene en este capítulo que uno de los motivos de este fracaso es que las políticas y acciones implementadas se han inspirado en el paradigma dominante de la sociedad de la información que se orienta por valores y prioridades que no coinciden con las del desarrollo de estas sociedades.

Teorías "proféticas" y "apologéticas" han exacerbado los beneficios de las TIC y han descrito a la SIC como una sociedad ho- 
mogénea, suponiendo que la extensión del uso de las TIC a toda la población permitirá lograr o mejorar el bienestar y el desarrollo social. Este discurso dominante justifica un proyecto de desarrollo que expresa una ola modernizadora. Una concepción con fuertes tendencias homogeneizantes, progresivas y lineales de los procesos, e idénticas para todas las sociedades independientemente de sus características específicas -ubicación estructura socio-económica, geográfica, cultura, actores, etc.-, se identifica con el proyecto de desarrollo de los países ricos que legitima la competitividad y la hegemonía mundial de estos en los sectores de punta del desarrollo tecnológico (Dubois y Cortés, 2005).

Las TIC aparecen en el centro de este modelo tecnocrático, como un modelo de desarrollo universal hegemónico. Algunos de los principios de este paradigma son: las telecomunicaciones benefician a la sociedad y a la economía; las TIC mejoran la relación costo-beneficio en el conjunto de la producción social; el desarrollo de las TIC y la universalización del conocimiento mejoran la distribución y el acceso a la información y por lo tanto a los beneficios económicos; su aplicación facilita el cambio social y mejores condiciones de vida (Cortés y Dubois, 2005).

Sin embargo, las desigualdades sociales también están presentes en la SIC a la vez que surgen una diversidad de modalidades de asumir esta etapa del desarrollo según las condiciones de los países, sus capacidades y su historia específica.

La brecha digital es uno de los conceptos de referencia. Este mismo concepto surge a partir de una apreciación que se enmarca en el paradigma dominante y que consiste en cuantificar el acceso a las TIC mediante indicadores de conectividad y de infraestructura. Se ha avanzado hacia significados menos tecnologicistas y más complejos, donde son consideradas otras variables y procesos. Así mismo está siendo cuestionada la relación lineal entre TIC y desarrollo, abordándola más como un vínculo de carácter recursivo, pues en la medida que por un lado refleja la brecha digital como una consecuencia de otras brechas de desarrollo 
Las políticas para la Sociedad de la Información y el Conocimiento en América Latina desde una mirada tecnologicista a un enfoque para el complejo

también esta contribuye a estas otras brechas (Gascó; Ezquiza; Acevedo; 2007). En la medida que se reduzca la brecha digital también se estará contribuyendo a la reducción de las otras.

De acuerdo a lo presentado hasta aquí son necesarias intervenciones y acciones explícitas y con un firme propósito de desarrollo. Las políticas se evidencian como un factor central en términos de la orientación y aprovechamiento de los beneficios de los adelantos tecnológicos para el desarrollo. Se considera que las TIC, en sí mismas no marcan la orientación del desarrollo, sino que más bien su uso debe estar orientado para el desarrollo y las prioridades específicas del mismo en los distintos contextos. En el marco de la elaboración de políticas para la SIC en las que las TIC contribuyan al desarrollo, es que se constata la confrontación entre paradigmas o miradas distintas sobre la SIC, muchas veces implícitas en las disyuntivas de los decisores. Es fundamental, retomar algunas consideraciones acerca de las principales características de estas políticas y de las críticas a las que hasta ahora se han implementado. A partir de esta revisión se podrán considerar nuevas dimensiones o preguntas a tener en cuenta para la definición e implementación de un nuevo tipo de políticas TIC orientadas al desarrollo.

Las estrategias de desarrollo pueden articularse de distinta forma con la SIC y que esto depende de las condiciones de cada país y de las características y la intervención de sus actores e instituciones (Castells y Himanen, 2003). Las TIC permiten desarrollar nuevas capacidades para alcanzar metas y objetivos con sentido para la vida de las personas, pero estos resultados no son espontáneos o inherentes a ellas. Es necesario instrumentar acciones y políticas que promuevan la utilización de las tecnologías para la ampliación de los derechos y oportunidades para el ejercicio de la libertad y la expansión de las capacidades de las personas (Gascó, 2007).

Influidos por la visión dominante, muchos debates sobre la orientación y énfasis de las políticas han puesto más énfasis en la 
dinámica de los mercados, los procedimientos de la gobernanza y regulación de las TIC y los servicios. En este marco se da por supuesto que el beneficio para los ciudadanos es directo y se trata sólo de brindarles acceso. Sin embargo, como sostiene Mansell, no hay evidencia de que una vez conectados los ciudadanos se empoderen como para llevar adelante la vida con un sentido deseado. Es importante construir una aproximación a las políticas para estos medios de comunicación, que implique la creación de capacidades pues constituyen derechos de los ciudadanos (Mansell, 2002).

En una revisión de las políticas nacionales de TIC en los países en desarrollo, Currie (S/F) encuentra distintos tipos de iniciativas en base a las cuales realiza una tipología. Sostiene que las primeras experiencias son de los ochenta, pero es recién en los noventa que hay una explosión y consolidación de las mismas. Muchas de estas no están claramente diferenciadas de sector de TIC y de las políticas de telecomunicaciones y en la mayor cantidad de casos no hay una conexión explícita con las metas nacionales de desarrollo. Todas varían en términos de sus objetivos, diseños e implementación, sin embargo encuentra dos diferenciaciones claras:

1) las que conciben a las TIC como un sector productivo y se orientan a fortalecer la industria y pueden estar orientadas al mercado de exportaciones o al desarrollo de capacidades nacionales, $\mathrm{y}$

2) las que consideran las TIC como habilitadoras de desarrollo socio-económico que a su vez pueden estar orientadas hacia el posicionamiento global o hacia objetivos de desarrollo. Estos tipos de políticas y estrategias no son excluyentes, pero tampoco necesariamente complementarios. Concluye según los estudios de caso realizados, es fundamental que las estrategias nacionales estén explícitamente enfocadas al desarrollo.

Finalmente, resalta la importancia de un marco para el despliegue de las TIC para el desarrollo que priorice las intervencio- 
Las políticas para la Sociedad de la Información y el Conocimiento en América Latina desde una mirada tecnologicista a un enfoque para el complejo

nes en TIC, identifique sinergias, maximice los impactos en el desarrollo y coordine las acciones de todos los actores participantes (Currie, s/f).

\section{Nuevas visiones y perspectivas para las políticas}

En los ámbitos de políticas TIC en América Latina se constatan distintas visiones y valores que fundamentan la orientación que deben tener las políticas para la SIC y sobre TIC en particular. En un enfoque tecnologicista se da prioridad a la infraestructura, la conectividad y esto es primordialmente medido a través de indicadores cuantitativos. En el caso del enfoque de mercado las acciones tenderán a ser delegadas a la dinámica del mismo y quedará en manos de los actores privados -por ejemplo las compañías de telecomunicaciones- la decisión, brindar acceso a las TIC. En un enfoque complejo se considerarán factores tales como el nivel educativo, la participación de los distintos actores involucrados y la ciudadanía en general, y se centrará en las transformaciones sociales y mejora de la calidad de vida que la utilización de las TIC pueda generar o potenciar desarrollo. Hasta ahora parecen haber predominado las políticas de conectividad e infraestructura y la preponderancia de indicadores cuantitativos. Se hacen por tanto necesarias las políticas en la SIC para el desarrollo que sean opuestas a la lógica excluyente del mercado y que estén enmarcadas en una estrategia de desarrollo y a partir de actores concretos (Rivoir, 2005).

Es el enfoque tecnologicista que ha predominado las políticas para la SIC en América Latina, y por tanto se han concentrado, desde los noventa a la primeros años del milenio, es mejorar la infraestructura y el acceso a las TIC. Si bien esto es imprescindible, es igualmente insuficiente para contribuir a los procesos de desarrollo pues sus logros se reducen a crear mercados y consumidores (Afonso, 2006; Finquelievich, 2003).

En otras investigaciones sobre usos de Internet en América Latina también se resaltaba el predominio de una perspectiva 
"instrumental" y "técnica". Se criticaba esta perspectiva en tanto limita y va en detrimento del potencial de Internet como lenguaje y sistema de representaciones a través del cual los jóvenes y los ciudadanos crean y recrean los relatos, las visiones de sí mismos y de la sociedad. Estas formas reduccionistas de visualizar las TIC, van en desmedro de su potencial para desarrollar nuevas relaciones, pedagogías, procesos comunicativos y formas de aprendizaje, pues impide al ciudadano empoderarse y apropiarse de esta herramienta. Es importante entender el uso de Internet en el contexto de los cambios culturales, las relaciones de poder y los cambios en los sistemas simbólicos y de circulación de conocimiento. Para ello es necesario innovar en los enfoques, metodologías y pedagogías que habiliten su uso social (Bonilla y Cliche, 2001).

Para desarrollar cambios en cuanto a políticas de uso de Internet, destacan Bonilla y Cliche (2001) que son necesarios tres procesos convergentes. Por un lado, la construcción de una nueva visión y proyectos que en la práctica promuevan el uso y apropiación de las TIC como formas de integración social, de desarrollo de nuevas pedagogías más participativas y horizontales. En segundo lugar, consolidar el Derecho a la Comunicación y la Cultura y a Internet y en tercer lugar, la creación de alianzas estratégicas de actores para el desarrollo social de las TIC.

Poniendo énfasis en los procesos y las metodologías, otros señalan que no se trata de que exista disponibilidad de servicios y contenidos "para" la población. Los enfoques definidos "desde arriba" reproducen las desigualdades y las brechas. Por tanto, los propios objetos tecnológicos deben ser objetos de intervención por parte de quienes lo utilizan. Araya considera que es necesario "fortalecer el dominio público mediante la creación de bienes colectivos globales que garanticen el acceso universal a la información y la comunicación"; "fomento de redes y colectivos electrónicos sin fines de lucro que aseguren la producción y circulación abierta, plural y diversa de información pública y contenido re- 
Las políticas para la Sociedad de la Información y el Conocimiento en América Latina desde una mirada tecnologicista a un enfoque para el complejo

levantes para el desarrollo humano"; "promoción de iniciativas de participación "desde" y "hacia" la comunidad que incluyan el uso tradicional e innovador de la comunicación a nivel local"; acciones educativas y de capacitación para el uso y apropiación social que contribuyan a desarrollar nuevos imaginarios tecnológicos y capacidades sociales desde identidades y perspectivas culturales, locales, regionales y nacionales, así como el fomento al desarrollo de tecnologías alternativas (Araya, 2003, p. 4-5).

Por su parte, Finquelievich sostenía ya hace unos años, la necesidad de cumplir con ciertos ejes para que las políticas TIC estén orientadas al desarrollo. Por un lado que las políticas y estrategias fueran multiactorales (multistakeholder) y que brinden servicios sociales de desarrollo sustentable, social y político. Por otra parte, brindar un acceso físico y también cultural, que implique un aprendizaje para la creación de contenidos propios. Sostenía que para ello es necesaria la creación de carreras para la SI y la producción y construcción de industrias tecnológicas nacionales y regionales. Por último, todos estos aspectos necesariamente atravesados por ejes de inclusión social, de género y minorías. En esta propuesta se integran distintos elementos que tienen que ver con los usos de las TIC, pero también con elementos de carácter macro o estructurales, sin olvidar las características de los procesos de cambio (Finquelievich, 2000).

Más recientemente otros enfoques han agregado complejidad a la temática agregando nuevas dimensiones a ser consideradas por las políticas. El Informe de Desarrollo Humano de Chile 2006, por ejemplo, sigue reforzando y confirmando que cuando las TIC constituyen un fin en sí mismo para las políticas, no se generan necesariamente oportunidades para el desarrollo. Revela que los usuarios, individuales y colectivos, deben disponer de condiciones objetivas y subjetivas dentro de las que se cuenta la capacidad para definir reflexivamente los fines del uso. En tal sentido, las TIC deben estar subordinadas a fines explicitados y éticamente legítimos, que guíen su uso concreto (PNUD, 2006). 
A los efectos de mejorar las políticas es fundamental reconocer las múltiples dimensiones de la brecha digital. Asimismo, es necesario saber para qué usar las TIC pues estas sirven a propósitos que van más allá de las técnicas y por lo tanto, también requiere que estas sean adaptadas a otros propósitos. En otro orden, promover regulaciones que resguarden los derechos de las personas de los usos nocivos, de la dependencia, la violación de la privacidad o para el control de las personas constituye pilares importantes de estas políticas. Finalmente, se destaca la importancia de utilizar las TIC para un nuevo relacionamiento en red a través de la digitalización debe buscar mejorar la participación y la ciudadanía. El Informe finaliza apelando a la necesidad de situar a la sociedad y a las personas en el centro del cambio tecnológico (PNUD, 2006).

En el mismo sentido, el informe del Global Information Society Watch 2007 - GISW (APC, 2007) señala la importancia de brindar el acceso universal a través de las políticas pues el mercado resulta insuficiente. A tales efectos son fundamentales nuevas políticas TIC para el desarrollo. Las principales deficiencias detectadas tienen que ver con que muchos países carecen de una visión de las TIC para su futuro, lo que a su vez impacta negativamente sobre aspectos tales como los de género, contenidos locales, derechos ciudadanos, o de apoyo a personas con capacidades diferentes. Concluye que es necesario que los gobiernos acepten que las políticas públicas de TIC para el desarrollo humano constituyen una inversión esencial.

En este marco, una deficiencia detectada es la falta de capacidad institucional que se suma a la falta de capacitación, habilidades y conciencia y que finalmente redunda en la reducción de la capacidad de actuar. Esto resulta en un pésimo entorno para la elaboración de las políticas de TIC y deriva en la fragmentación de iniciativas y de las propias políticas. Es cierto que en otros países constatan la existencia de políticas pero estas no asumen una perspectiva de desarrollo. Finalmente, se señala también que 
Las políticas para la Sociedad de la Información y el Conocimiento en América Latina desde una mirada tecnologicista a un enfoque para el complejo

muchos procesos participativos y documentados, acciones y acumulación de aprendizajes, se ven desperdiciados por el cambio de las autoridades de gobierno. Es decir, la sustentabilidad de los procesos interesantes e importantes en términos del desarrollo.

\section{Conclusiones}

Los elementos analizados dan cuenta de las dificultades que ha experimentado el continente latinoamericano para elaborar políticas TIC orientadas al desarrollo. Se tomado conciencia por parte de los decisores públicos acerca de la importancia de estas tecnologías y se han desarrollado experiencias de políticas públicas a nivel de los distintos países. Sin embargo, la mera existencia de políticas no es suficiente para que estas se relacionen a procesos de desarrollo. Es necesario que estas asuman características específicas. En primer lugar, que se enmarquen en un paradigma complejo abandonando todo el sesgo tecnologicista.

Los procesos también son importantes y por eso las características de las acciones deben ser especialmente atendidas. $\mathrm{Si}$ bien se revelan ciertas coincidencias en las críticas a las políticas implementadas en términos de estar ligadas al paradigma dominante, también surgen propuestas respecto de ejes y metodologías a abordar. A nivel discursivo es probable que se encuentren confluencias en torno a objetivos para el desarrollo, y valores considerados dentro de los derechos humanos. No obstante, es necesario que el diseño y la implementación de las políticas deben ser coherentes con el nuevo paradigma. La concepción de derechos humanos y construcción de ciudadanía que implique un control sobre los medios y recursos que implican estas tecnologías constituyen parte de los fundamentos a asumir por estas iniciativas. Asimismo, y sin dejar de lado los objetivos de acceso y conectividad, deben ir acompañados de la búsqueda de apropiación y uso con sentido para el desarrollo de las personas y las comunidades. 


\section{Referencias bibliográficas}

Afonso, Carlos. Desenvolvimento humano e apropriacao das TICs. Ritla, Abril, 2006.

Araya, Rubén (2003). “Comunidades y portales ciudadanos: ¿Para qué? Reflexiones desde una visión social sobre Internet". http:/ / redistic.org/brecha/es/17_-_Rub\%E9n_Araya.html

Arocena, Rodrigo y Judith. Sutz Subdesarrollo e Innovación. Navegando contra el viento Cambridge University Press/OEI, Madrid, 2003.

Association for Progressive Comunications (APC); Third World Institute (ITeM) "Global Information Society Watch 2007. Focus on Participation", 2007.

Bonilla, Marcelo y Gilles Cliche. "Internet y sociedad en América Latina". Introducción. Fundación Acceso. Costa Rica. 2001.

Currie, Willie. "National Approaches to ICT". http://wsispapers.choike. org/national_approaches_ict.pdf

Castells, Manuel. La era de la información. Economía, Sociedad Cultura. Vol. I La sociedad red. Alianza Editorial, Barcelona. 2000.

Castells, Manuel y Pekka Himanen. El Estado de Bienestar y la Sociedad de la Información. El modelo finlandés. Alianza Editorial. Madrid, 2002.

Finquelievich, Susana. Indicadores de la Sociedad de la Información en Educación, Ciencia, Cultura, Comunicación e Información, en ALC. RICYT/OCT, Lisboa, 2003. http://www.itu.int/dms_pub/itus/md/03/wsispc2/doc/S03-WSISPC2-DOC-0007!!MSW-S.doc

Finquelievich, Susana (2000). “TIC, desarrollo y reducción de la pobreza en América Latina y el Caribe". http://www.links.org.ar/infoteca/ticsypobrezalac.rtf

Gascó-Hernández, Mila; Fran Equiza-López y Manuel Acevedo-Ruiz. Information Communication Technologies and Human Development: Opportunities and Challenges IIG, IGI Publishing 2007.

Mansell, Robin (2002). "From Digital Divides to Digital Entitlements in Knowledge Societies". Current Sociology, Vol. 50, No. 3, 407426. http:/ / csi.sagepub.com/cgi/content/abstract/50/3/407

Mattelart, Armand. Historia de la sociedad de la Información. Paidós Comunicación 132. 2002. 
Las políticas para la Sociedad de la Información y el Conocimiento en América Latina desde una mirada tecnologicista a un enfoque para el complejo

PNUD. Informe sobre Desarrollo Humano en Chile 2006. “Las nuevas tecnologías: ¿un salto al futuro?" PNUD, Santiago, 2006.

Rivoir, Ana. "The information and Knowledge Society in Latin America and the Caribbean: Different Approaches an their implications for Policies". En: Information Society for the South: Vision or Hallucination? Instituto del Tercer Mundo. Montevideo, 2005. 